Errata : The following numerical values should be quadrupled.

p.14, II . $1: 6.25$ and 15.7895

p.14, II . 3: 12.7232

p.16, I . $6: 11.3258$

p.15 Fig. $3:$ vertical scale and 12.7232 in the caption

p.16 Fig. 4 : vertical scale and 11.3258 in the caption

The authors acknowledge Ms.Dora Ludvig and Dr. Péter Csóka for pointing out the errors. 


\title{
Bounding Contingent Claim Prices via Hedging Strategy with Coherent Risk Measures
}

\author{
Jun-ya Gotoh* $\quad$ Yoshitsugu Yamamoto ${ }^{\dagger} \quad$ Weifeng Yao ${ }^{\ddagger}$
}

April 2011

communicated by David G. Luenberger

\begin{abstract}
We generalize the notion of arbitrage based on the coherent risk measure, and investigate a mathematical optimization approach for tightening the lower and upper bounds of the price of contingent claims in incomplete markets. Due to the dual representation of coherent risk measures, the lower and upper bounds of price are located by solving a pair of semi-infinite linear optimization problems, which further reduce to linear optimization when conditional value-at-risk $(\mathrm{CVaR})$ is used as risk measure. We also show that the hedging portfolio problem is viewed as a robust optimization problem. Tuning the parameter of the risk measure, we demonstrate by numerical examples that the two bounds approach to each other and converge to a price that is fair in the sense that seller and buyer face the same amount of risk.
\end{abstract}

Keywords. incomplete market, option price bounds, coherent risk measures, hedging strategy, semi-infinite linear optimization

AMS Classification 2010. 49M29, 90C34, 91B24, 91G20.

\section{Introduction}

The pricing theory of financial contingent claims has an illustrious history which started in 1973 when Black and Scholes presented the option pricing model, and then Merton extended their model in several important ways. Since their seminal works, numerous researchers have committed to establish the mathematical foundation for the pricing of contingent claims by modeling the uncertainty of asset market in either of two ways: continuous models and discrete models. The former assumes that investors can trade assets in a market at any moment and there are infinitely many possible states, while the latter assumes that trade can be made only at finite times, and the number of possible states is usually finite.

As in the Black-Scholes model, continuous models provide an explicit formula of pricing by assuming that the underlying asset price follows a specific stochastic process such as geometric Brownian motion.

\footnotetext{
*Department of Industrial and Systems Engineering, Chuo University, 2-13-27 Kasuga, Bunkyo-ku, Tokyo 112-8551, Japan, e-mail: jgoto@indsys.chuo-u.ac.jp. Partly supported by MEXT Grant-in-Aid for Young Researchers (B) 20710120. The corresponding author.

${ }^{\dagger}$ Graduate School of Systems and Information Engineering, University of Tsukuba, Tsukuba, Ibaraki 305-8573, Japan, e-mail: yamamoto@sk.tsukuba.ac.jp. Partly supported by JSPS Grant-in-Aid for Scientific Research (C) 22510136

$\ddagger$ e-mail: wf_yao@hotmail.com.
} 
However, the assumption is often too restrictive to apply the model to real market data. In addition, most of the models can treat only a small number of underlying assets simultaneously.

In order to fit the model to real situations, several directions have been considered. One direction is to introduce a general class of stochastic processes such as Lévi process (e.g., [1]) and stochastic volatility model (e.g., [2]). Another direction is to abandon the use of parametric distributions, for example, by supposing that only partial information of the distribution is available. Several researches $[3,4,5,6]$ provide convex optimization approaches to bounding the price of European-type contingent claims when moments are the sole information available about the underlying asset price distribution.

On the other hand, discrete model can deal with general distributions with the aid of scenario tree approximation. Cox, Ross and Rubinstein model [7] and several interest rate tree models such as Hull [8] are well-known examples. It can also treat multiple underlying assets (e.g., [9, 10]). Therefore, it forms the basis for numerically computing the price of contingent claims such as exotic options on multiple underlying assets. Furthermore, discrete model can treat the valuation of a contingent claim in incomplete markets in a similar manner as in the complete market.

Linear optimization has played a vital role in the development of discrete models. For example, Ritchken [11] formulates the problem of computing upper and lower bounds of the price of a Europeantype contingent claim as a linear optimization problem; introducing the risk attitude of investors, Ritchken, Kuo, Basso and Pianca [12,13] extend the approach so as to reduce the gap of the pricing bounds. King and his coauthor $[10,14]$ point out, nevertheless, that such a reduction does not explain pricing mechanism unless the gap vanishes since neither buyer nor seller would be motivated to make a deal in the presence of gap.

In this paper, we replace the traditional no-risk condition with an alternative condition associated with a coherent risk measure, and generalize the fundamental theory of asset pricing of discrete models in King [10]. As for the continuous models, this direction of generalization has been discussed in the literature, e.g., $[15,16]$. Our work is characterized by the full use of the duality theory of mathematical optimization, especially of semi-infinite linear optimization. We then present a parametric optimization method for narrowing and hopefully closing the gap between the upper and lower bounds, which would be a promising way of pricing contingent claims in incomplete markets. It is worth mentioning that, when the conditional value-at-risk ( $\mathrm{CVaR})[17]$ is used as the risk measure, the resulting problem is a linear optimization problem. We finally show that computing the price bounds based on a coherent risk measure links to a robust optimization, where underlying probability plays a role of nominal vector for 
uncertainty set of probability measures. See, e.g., [18] for a survey of robust optimization.

In next section, we briefly review the classic and well-known results about the no-arbitrage option pricing in a discrete market model. In Section 3, we introduce a generalized notion of arbitrage based on coherent risk measures. By exploiting the duality theory for semi-infinite linear optimization, a generalized version of the fundamental theorem of asset pricing in discrete market model is provided. Section 4 is devoted to computing the upper and lower bounds of the price of a European-type contingent claim. In Section 5, some numerical examples are given for illustrating how to close the gap between two bounds. Section 6 concludes the paper with some remarks. Some lemmas will be proved in Appendix.

\section{Pricing Bounds and Arbitrage Opportunity}

We briefly review the methods in which mathematical optimization techniques are used for computing the upper and lower bounds of the price of contingent claims.

\subsection{Notations and Basic Assumptions}

We first introduce a scenario tree which describes the uncertainty of contingent claims' values according to King [10].

We denote the depth of the scenario tree by $T$ and write $[T]:=\{0,1, \ldots, T\}$. Let $\mathcal{N}_{t}$ be the set of all nodes at depth $t$ in the scenario tree for $t \in[T]$, and let $\mathcal{N}$ be the set of all nodes, i.e., $\mathcal{N}:=\bigcup_{0 \leq t \leq T} \mathcal{N}_{t}$. We denote the number of nodes of $\mathcal{N}_{t}$ by $n_{t}$ in lower case. The set $\mathcal{N}_{0}$ consists of the initial node 0 alone, i.e., $n_{0}=1$. We abbreviate $\mathcal{N} \backslash \mathcal{N}_{t}$ to $\mathcal{N}_{-t}$. In the scenario tree, every node $n \in \mathcal{N}_{t}$ for $t=1,2, \ldots, T$ has a unique parent node in $\mathcal{N}_{t-1}$ denoted by $a(n)$, and every node $n \in \mathcal{N}_{t}$ for $t=0,1, \ldots, T-1$ has a nonempty set of child nodes in $\mathcal{N}_{t+1}$, denoted by $c(n)$. The original probability distribution $\boldsymbol{p}$ is modeled by attaching a positive weight $p_{n}$ to each terminal node $n \in \mathcal{N}_{T}$ so that $\sum_{n \in \mathcal{N}_{T}} p_{n}=1$. Each nonterminal node is given the probability $\tilde{p}_{n}$ defined inductively by $\tilde{p}_{n}:=\sum_{m \in c(n)} \tilde{p}_{m}$, where $\tilde{p}_{n}:=p_{n}$ for $n \in \mathcal{N}_{T}$.

We suppose that the market has $J+1$ tradable securities indexed by $j=0, \ldots, J$ whose prices at node $n$ are denoted by the vector $\boldsymbol{s}_{n}:=\left(s_{n}^{0}, s_{n}^{1}, \ldots, s_{n}^{J}\right)^{\top}$. We assume that the security 0 , which we refer to as numeraire, always has a positive value, i.e., $s_{n}^{0}>0$ for all $n \in \mathcal{N}$. Let us denote the discount rate by $\delta_{n}:=1 / s_{n}^{0}$ and $\boldsymbol{z}_{n}:=\delta_{n} \boldsymbol{s}_{n}$. Then the vector $\boldsymbol{z}_{n}$ is the relative price vector with respect to the numeraire. Obviously, $z_{n}^{0}=1$, for all $n \in \mathcal{N}$.

Note that we can develop the argument without introducing the notion of random variable because any random variable in the tree can be represented by a finite dimensional vector attached with a node. 
For convenience, we reserves the upper case characters for denoting random variables. For $t \in[T]$, we denote the random variable vector on $\mathcal{N}_{t}$ with $\boldsymbol{Z}_{t}(n)=\boldsymbol{z}_{n}$ for each $n \in \mathcal{N}_{t}$ by $\boldsymbol{Z}_{t}$.

\subsection{Fundamental Theorem of Asset Pricing}

For $j=0,1, \ldots, J$ and $n \in \mathcal{N}$, let $\theta_{n}^{j}$ denote the amount of security $j$ held by the investor at node $n$, and let $\boldsymbol{\theta}_{n}:=\left(\theta_{n}^{0}, \theta_{n}^{1}, \ldots, \theta_{n}^{J}\right)^{\top}$. We call $\left\{\boldsymbol{\theta}_{n} \mid n \in \mathcal{N}\right\}$ a trading strategy. We sometimes denote it simply by $\theta$.

Definition 2.1. We say that a trading strategy $\boldsymbol{\theta}$ is self-financing iff it satisfies

$$
\boldsymbol{z}_{n}^{\top}\left(\boldsymbol{\theta}_{n}-\boldsymbol{\theta}_{a(n)}\right)=0, \quad\left(n \in \mathcal{N}_{-0}\right)
$$

The traditional arbitrage strategy is defined as follows.

Definition 2.2 (Traditional Arbitrage Strategy). Arbitrage is a self-financing trading strategy $\boldsymbol{\theta}$ that begins with zero initial value at time 0 , maintains a non-negative value at each terminal node $n \in \mathcal{N}_{T}$ and has a positive expected value at the maturity date $T$. Mathematically, arbitrage is a trading strategy $\boldsymbol{\theta}$ that is defined by

$$
\begin{array}{ll}
\boldsymbol{z}_{0}^{\top} \boldsymbol{\theta}_{0}=0, & \\
\boldsymbol{z}_{n}^{\top}\left(\boldsymbol{\theta}_{n}-\boldsymbol{\theta}_{a(n)}\right)=0, & \left(n \in \mathcal{N}_{-0}\right), \\
\boldsymbol{z}_{n}^{\top} \boldsymbol{\theta}_{n} \geq 0, & \left(n \in \mathcal{N}_{T}\right), \\
\sum_{n \in \mathcal{N}_{T}} p_{n} \boldsymbol{z}_{n}^{\top} \boldsymbol{\theta}_{n}>0 . &
\end{array}
$$

The market is said to be arbitrage free iff there is no chance of arbitrage in the market. Harrison and Kreps [19] proved that the absence of arbitrage is essentially equivalent to the existence of an equivalent martingale probability measure $\tilde{\boldsymbol{q}}$ such that

1) $\tilde{\boldsymbol{q}}$ agrees with $\tilde{\boldsymbol{p}}$ on impossible events, i.e., $\tilde{q}_{n}=0$ if and only if $\tilde{p}_{n}=0$, for all $n \in \mathcal{N}$,

2) $\left\{\boldsymbol{Z}_{t} \mid t \in[T]\right\}$ is a martingale process under the probability measure $\tilde{\boldsymbol{q}}$, i.e.,

$$
\tilde{q}_{n} \boldsymbol{z}_{n}=\sum_{m \in c(n)} \tilde{q}_{m} \boldsymbol{z}_{m}, \quad\left(n \in \mathcal{N}_{-T}\right) .
$$

Further, the no arbitrage price of security $Y$ is given by the expected value of $Y$ at the maturity date $T$ based on an arbitrarily chosen equivalent martingale probability measure. 
Theorem 2.1 (Fundamental Theorem of Asset Pricing).

1) There is no arbitrage if and only if there is a martingale probability measure $\tilde{\boldsymbol{q}}$ equivalent to $\tilde{\boldsymbol{p}}$.

2) In the arbitrage free market, the lower and upper bounds of the price of a contingent claim $Y$ are given, respectively, by

$$
\frac{1}{\delta_{0}} \min _{\tilde{\boldsymbol{q}} \in \mathcal{M}} \sum_{n \in \mathcal{N}_{T}} \tilde{q}_{n} \delta_{n} y_{n} \quad \text { and } \quad \frac{1}{\delta_{0}} \max _{\tilde{\boldsymbol{q}} \in \mathcal{M}} \sum_{n \in \mathcal{N}_{T}} \tilde{q}_{n} \delta_{n} y_{n}
$$

where $\mathcal{M}$ is the set of martingale probability measures, and $y_{n}$ is the future value of contingent claim $Y$ at node $n$.

Proof. See Theorem 1 of [10], for example.

If the price of contingent claim $Y$ is less than or equal to the lower bound in Theorem 2.1, there exists an arbitrage for buyer; on the other hand, a price which is greater than or equal to the upper bound induces an arbitrage for seller. Thus, the interval between the two bounds in the theorem is called the no arbitrage interval of $Y$.

\section{Acceptance Set, Coherent Risk Measure and Arbitrage}

We introduce concepts of the acceptance set and coherent risk measure after the fashion of Artzner et al. [20], and then describe a generalization of the arbitrage. The condition for the absence of arbitrage based on the coherent risk measure is also given.

\subsection{Generalization of Arbitrage and Relation to Coherent Measure}

We define the acceptance set of the investor by the set of net worths of a contingent claim that the investor is willing to take, and denote it by $\mathcal{A}$. Given an acceptance set $\mathcal{A}$, a trading strategy $\boldsymbol{\theta}$ is said to be acceptable iff $\left(\boldsymbol{z}_{n}^{\top} \boldsymbol{\theta}_{n}\right)_{n \in \mathcal{N}_{T}} \in \mathcal{A}$.

Definition 3.1 (Generalized Arbitrage). A self-financing trading strategy $\boldsymbol{\theta}$ is said to be a generalized arbitrage associated with $\mathcal{A}$ iff it begins with zero initial value, maintains an acceptable net worth at each terminal node $n \in \mathcal{N}_{T}$, and has a positive expected value at the maturity date $T$. Mathematically, the 
generalized arbitrage associated with an acceptance set $\mathcal{A}$ is a trading strategy $\boldsymbol{\theta}$, such that

$$
\begin{aligned}
& \boldsymbol{z}_{0}^{\top} \boldsymbol{\theta}_{0}=0, \\
& \boldsymbol{z}_{n}^{\top}\left(\boldsymbol{\theta}_{n}-\boldsymbol{\theta}_{a(n)}\right)=0, \quad\left(n \in \mathcal{N}_{-0}\right), \\
& \left(\boldsymbol{z}_{n}^{\top} \boldsymbol{\theta}_{n}\right)_{n \in \mathcal{N}_{T}} \in \mathcal{A}, \\
& \sum_{n \in \mathcal{N}_{T}} p_{n} \boldsymbol{z}_{n}^{\top} \boldsymbol{\theta}_{n}>0 .
\end{aligned}
$$

Note that the arbitrage associated with

$$
\mathbb{R}_{+}^{n_{T}}:=\left\{\boldsymbol{x} \in \mathbb{R}^{n_{T}} \mid \boldsymbol{x} \geq \mathbf{0}\right\}
$$

coincides with the traditional arbitrage in Definition 2.2. If $\mathcal{A}$ contains $\mathbb{R}_{+}^{n_{T}}$, the no-risk condition for the arbitrage associated with $\mathcal{A}$ is weaker than that for the traditional arbitrage.

Definition 3.2 (Acceptance Set associated with a Risk Measure [20]). Given a risk measure $\rho$, the acceptance set associated with $\rho$, denoted by $\mathcal{A}_{\rho}$, is defined by

$$
\mathcal{A}_{\rho}:=\left\{\boldsymbol{x} \in \mathbb{R}^{n_{T}} \mid \rho(\boldsymbol{x}) \leq 0\right\} .
$$

Definition 3.3 (Coherent Risk Measure [20]). A risk measure $\rho$ is said to be coherent iff $\rho$ has the following four properties.

1) Translation invariance: For all $\boldsymbol{x}$ and real number $c, \rho(\boldsymbol{x}+c \boldsymbol{e})=\rho(\boldsymbol{x})-c$.

2) Subadditivity: For all $\boldsymbol{x}_{1}$ and $\boldsymbol{x}_{2}, \rho\left(\boldsymbol{x}_{1}+\boldsymbol{x}_{2}\right) \leq \rho\left(\boldsymbol{x}_{1}\right)+\rho\left(\boldsymbol{x}_{2}\right)$.

3) Positive homogeneity: For all $\lambda \geq 0$ and net worth $\boldsymbol{x}, \rho(\lambda \boldsymbol{x})=\lambda \rho(\boldsymbol{x})$.

4) Monotonicity: For all $\boldsymbol{x}$ and $\boldsymbol{y}$ with $\boldsymbol{x} \leq \boldsymbol{y}$, it holds that $\rho(\boldsymbol{y}) \leq \rho(\boldsymbol{x})$.

Artzner et al. [20] proved that, if $\rho$ is a coherent risk measure, then the acceptance set $\mathcal{A}_{\rho}$ has the following properties and vice versa.

1) $\mathcal{A}_{\rho}$ is a closed convex cone.

2) $\mathbb{R}_{+}^{n_{T}} \subseteq \mathcal{A}_{\rho}$ and $\mathcal{A}_{\rho} \cap \mathbb{R}_{--}^{n_{T}}=\phi$, where $\mathbb{R}_{--}^{n_{T}}:=\left\{\boldsymbol{x} \in \mathbb{R}^{n_{T}} \mid \boldsymbol{x}<\mathbf{0}\right\}$.

Let $\mathbb{E}_{\boldsymbol{\pi}}(\boldsymbol{x})=\boldsymbol{\pi}^{\top} \boldsymbol{x}$. They also showed that if $\mathcal{P}_{\rho}$ is defined as

$$
\mathcal{P}_{\rho}:=\left\{\boldsymbol{\pi} \mid \boldsymbol{\pi} \text { is a probability measure on } \mathcal{N}_{T} \text { and } \mathbb{E} \boldsymbol{\pi}(-\boldsymbol{x}) \leq \rho(\boldsymbol{x}) \text {, for all } \boldsymbol{x} \in \mathbb{R}^{n_{T}}\right\},
$$


then $\rho(\cdot)$ satisfies

$$
\rho(\boldsymbol{x})=\sup \left\{\mathbb{E}_{\boldsymbol{\pi}}(-\boldsymbol{x}) \mid \boldsymbol{\pi} \in \mathcal{P}_{\rho}\right\} .
$$

We also see that $\rho\left(-\left(\boldsymbol{z}_{n}^{\top} \boldsymbol{\theta}_{n}\right)_{n \in \mathcal{N}_{T}}\right) \leq 0$ if and only if

$$
\sum_{n \in \mathcal{N}_{T}} \pi_{n} \boldsymbol{z}_{n}^{\top} \boldsymbol{\theta}_{n} \geq 0, \quad\left(\boldsymbol{\pi} \in \mathcal{P}_{\rho}\right) .
$$

Lemma 3.1. $\mathcal{P}_{\rho}$ is a compact convex subset of $\mathbb{R}^{n_{T}}$ that does not contain the origin.

Proof. By definition,

$$
\mathcal{P}_{\rho}=\bigcap_{\boldsymbol{x} \in \mathbb{R}^{n_{T}}}\left\{\boldsymbol{\pi} \mid-\boldsymbol{\pi}^{\top} \boldsymbol{x} \leq \rho(\boldsymbol{x})\right\} \cap\left\{\boldsymbol{\pi} \mid \boldsymbol{\pi} \geq \mathbf{0}, \boldsymbol{e}^{\top} \boldsymbol{\pi}=1\right\},
$$

all of which are closed convex sets, and the right most set is a compact set that does not contain the origin.

\subsection{Absence of Arbitrage}

We hereafter consider the acceptance set $\mathcal{A}_{\rho}$ defined by a coherent risk measure $\rho$. We define $\rho$-arbitrage as follows.

Definition 3.4 ( $\rho$-Arbitrage). For any coherent risk measure $\rho$, a $\rho$-arbitrage is a trading strategy $\boldsymbol{\theta}=\left(\boldsymbol{\theta}_{n}\right)_{n \in \mathcal{N}}$ that satisfies

$$
\begin{array}{ll}
\boldsymbol{z}_{0}^{\top} \boldsymbol{\theta}_{0}=0, & \\
\boldsymbol{z}_{n}^{\top}\left(\boldsymbol{\theta}_{n}-\boldsymbol{\theta}_{a(n)}\right)=0, & \left(n \in \mathcal{N}_{-0}\right), \\
\sum_{n \in \mathcal{N}_{T}} \pi_{n} \boldsymbol{z}_{n}^{\top} \boldsymbol{\theta}_{n} \geq 0, & \left(\boldsymbol{\pi} \in \mathcal{P}_{\rho}\right), \\
\sum_{n \in \mathcal{N}_{T}} p_{n} \boldsymbol{z}_{n}^{\top} \boldsymbol{\theta}_{n}>0 . &
\end{array}
$$

Note that the system (13), (14) and (15) is always consistent since $\boldsymbol{\theta}=(\mathbf{0})_{n \in \mathcal{N}}$ satisfies it. We say that the system satisfies the Slater constraint qualification when there is a vector $\hat{\boldsymbol{\theta}}=\left(\hat{\boldsymbol{\theta}}_{n}\right)_{n \in \mathcal{N}}$ satisfying

$$
\begin{array}{ll}
\boldsymbol{z}_{0}^{\top} \hat{\boldsymbol{\theta}}_{0}=0, & \\
\boldsymbol{z}_{n}^{\top}\left(\hat{\boldsymbol{\theta}}_{n}-\hat{\boldsymbol{\theta}}_{a(n)}\right)=0, & \left(n \in \mathcal{N}_{-0}\right), \\
\sum_{n \in \mathcal{N}_{T}} \pi_{n} \boldsymbol{z}_{n}^{\top} \hat{\boldsymbol{\theta}}_{n}>0, & \left(\boldsymbol{\pi} \in \mathcal{P}_{\boldsymbol{\rho}}\right) .
\end{array}
$$

Based on the above generalized notion of arbitrage, we show that the existence of a martingale measure is a necessary condition for the no $\rho$-arbitrage. For a probability measure $\tilde{\boldsymbol{q}}=\left(\tilde{q}_{n}\right)_{n \in \mathcal{N}}$ we denote its restriction to $\mathcal{N}_{T}$ by $\tilde{\boldsymbol{q}}_{T}=\left(\tilde{q}_{n}\right)_{n \in \mathcal{N}_{T}}$. 
Theorem 3.1. Suppose that system (13), (14) and (15) satisfy the Slater constraint qualification, and also suppose that $\boldsymbol{p} \in \mathcal{P}_{\rho}$. If there is no $\rho$-arbitrage, then there is a probability measure $\tilde{\boldsymbol{q}}$ such that

1) $\tilde{\boldsymbol{q}}_{T} \in \mathcal{P}_{\rho}$,

2) $\tilde{q}_{n}>0$ whenever $p_{n}>0$ for $n \in \mathcal{N}_{T}$,

3) $\left\{\boldsymbol{Z}_{t} \mid t \in[T]\right\}$ is a martingale process under $\tilde{\boldsymbol{q}}$.

Proof. Let us consider the optimization problem

$$
\begin{array}{|lll}
\inf & -\sum_{n \in \mathcal{N}_{T}} p_{n} \boldsymbol{z}_{n}^{\top} \boldsymbol{\theta}_{n}, & \\
\text { subject to } & \boldsymbol{z}_{0}^{\top} \boldsymbol{\theta}_{0}=0, \\
& \boldsymbol{z}_{n}^{\top}\left(\boldsymbol{\theta}_{n}-\boldsymbol{\theta}_{a(n)}\right)=0, \quad\left(n \in \mathcal{N}_{-0}\right), \\
& \sum_{n \in \mathcal{N}_{T}} \pi_{n} \boldsymbol{z}_{n}^{\top} \boldsymbol{\theta}_{n} \geq 0, \quad\left(\boldsymbol{\pi} \in \mathcal{P}_{\rho}\right) .
\end{array}
$$

Since $\mathcal{P}_{\rho}$ is compact, we see that the set of coefficient vectors $\left\{\left(\pi_{n} \boldsymbol{z}_{n}\right)_{n \in \mathcal{N}_{T}} \mid \boldsymbol{\pi} \in \mathcal{P}_{\rho}\right\}$ is compact. Suppose that there be no $\rho$-arbitrage, i.e., the optimal value of the problem be zero. Then, by Lemma A.3, its dual problem is feasible. That is,

$$
\begin{aligned}
& \lambda_{n} \boldsymbol{z}_{n}=\sum_{m \in c(n)} \lambda_{m} \boldsymbol{z}_{m}, \quad\left(n \in \mathcal{N}_{-T}\right), \\
& p_{n} \boldsymbol{z}_{n}=\lambda_{n} \boldsymbol{z}_{n}-\sum_{i \in I} \mu_{i} \pi_{n}^{(i)} \boldsymbol{z}_{n}, \quad\left(n \in \mathcal{N}_{T}\right),
\end{aligned}
$$

hold for some finite number of probability measures $\boldsymbol{\pi}^{(i)} \in \mathcal{P}_{\rho}(i \in I), \lambda_{n}(n \in \mathcal{N})$ and $\mu_{i} \geq 0(i \in I)$. Since $z_{n}^{0}=1$, for all $n \in \mathcal{N}$ it holds that

$$
\begin{array}{ll}
\lambda_{n}=\sum_{m \in c(n)} \lambda_{m}, & \left(n \in \mathcal{N}_{-T}\right), \\
\lambda_{n}=p_{n}+\sum_{i \in I} \mu_{i} \pi_{n}^{(i)}, & \left(n \in \mathcal{N}_{T}\right) .
\end{array}
$$

By using (20) and (21) repeatedly we see that

$$
\begin{aligned}
& \lambda_{n}>0 \text { when } \tilde{p}_{n}>0, \\
& \lambda_{0}=\sum_{n \in \mathcal{N}_{T}} \lambda_{n}=\sum_{n \in \mathcal{N}_{T}}\left(p_{n}+\sum_{i \in I} \mu_{i} \pi_{n}^{(i)}\right)=1+\sum_{i \in I} \mu_{i}>0 .
\end{aligned}
$$

Let $\tilde{q}_{n}$ be defined as

$$
\tilde{q}_{n}=\frac{\lambda_{n}}{\lambda_{0}} \quad(n \in \mathcal{N})
$$

and we see that $\tilde{\boldsymbol{q}}=\left(\tilde{q}_{n}\right)_{n \in \mathcal{N}}$ has the desired properties. Clearly, $\tilde{q}_{n}>0$ whenever $\tilde{p}_{n}>0$. By (18) and 
(20) we have

$$
\begin{array}{ll}
\tilde{q}_{n} \boldsymbol{z}_{n}=\sum_{m \in c(n)} \tilde{q}_{m} \boldsymbol{z}_{m}, & \left(n \in \mathcal{N}_{-T}\right), \\
\tilde{q}_{n}=\sum_{m \in c(n)} \tilde{q}_{m}, & \left(n \in \mathcal{N}_{-T}\right),
\end{array}
$$

which implies that $\left\{\boldsymbol{Z}_{t} \mid t \in[T]\right\}$ be a martingale process under $\tilde{\boldsymbol{q}}$.

For $n \in \mathcal{N}_{T}$, we have from (21) that

$$
\tilde{q}_{n}=\frac{\lambda_{n}}{\lambda_{0}}=\frac{1}{1+\sum_{i \in I} \mu_{i}} p_{n}+\sum_{i \in I} \frac{\mu_{i}}{1+\sum_{i \in I} \mu_{i}} \pi_{n}^{(i)} .
$$

Therefore $\tilde{\boldsymbol{q}}_{T}$ is a convex combination of $\boldsymbol{p}$ and $\boldsymbol{\pi}^{(i)}$ 's. Since all of those are in $\mathcal{P}_{\rho}$, we have by the convexity of $\mathcal{P}_{\rho}$ that $\tilde{\boldsymbol{q}}_{T}$ lies in $\mathcal{P}_{\rho}$. This proves the theorem.

The dual variables $\lambda_{n}$ play a similar role to $y_{n}$ in Theorem 1 of [10], in which $y_{n} \geq p_{n}$ for $n \in \mathcal{N}_{T}$ is shown. Thus, $\lambda_{n}$ can be considered as a strictly positive process similar to $y_{n}$ in [10]. Besides, $\mu_{n}$ play a role in enhancing the positivity of $\lambda_{n}$.

Theorem 3.2. Suppose that $\mathcal{P}_{\rho}$ be a polytope and $\boldsymbol{p} \in \mathcal{P}_{\rho}$. If there is no $\rho$-arbitrage, there is a probability measure $\tilde{\boldsymbol{q}}$ satisfying the same conditions as in Theorem 3.1 .

Proof. When $\mathcal{P}_{\rho}$ is a polytope, the infinitely many inequality constraints of (17) are equivalent to a finite number of inequality constraints each corresponding to an extreme point of $\mathcal{P}_{\rho}$. Then the LP duality in Lemma A.3 holds.

In the following we write $\Pi:=\left\{\boldsymbol{\pi} \mid \boldsymbol{\pi} \in \mathbb{R}^{n_{T}}, \boldsymbol{e}^{\top} \boldsymbol{\pi}=1\right\}$ and denote the interior of $\mathcal{P}_{\rho}$ relative to the hyperplane $\Pi$ by $\operatorname{ri}_{\Pi}\left(\mathcal{P}_{\rho}\right)$.

Theorem 3.3. If there is a martingale probability measure $\tilde{\boldsymbol{q}}$ such that $\tilde{\boldsymbol{q}}_{T} \in r i_{\Pi}\left(\mathcal{P}_{\rho}\right)$, then there is no $\rho$-arbitrage.

Proof. Since $\tilde{\boldsymbol{q}}_{T} \in \operatorname{ri}_{\Pi}\left(\mathcal{P}_{\rho}\right), \tilde{q}_{n}>0$ for all $n \in \mathcal{N}_{T}$ and there is a positive $\varepsilon$ such that the intersection of the hyperplane $\Pi$ and the $\varepsilon$-neighborhood of $\tilde{\boldsymbol{q}}_{T}$ is included in $\mathcal{P}_{\rho}$.

By Lemma A.4 we see that $\left(\alpha \tilde{\boldsymbol{q}}_{T}-\boldsymbol{p}\right) /\left\|\alpha \tilde{\boldsymbol{q}}_{T}-\boldsymbol{p}\right\|_{1}$ lies in this neighborhood for a sufficiently large $\alpha$, and hence in $\mathcal{P}_{\rho}$. Let $\tilde{\boldsymbol{r}}=(\alpha \tilde{\boldsymbol{q}}-\tilde{\boldsymbol{p}}) /\|\alpha \tilde{\boldsymbol{q}}-\tilde{\boldsymbol{p}}\|_{1}, \boldsymbol{\lambda}=\alpha \tilde{\boldsymbol{q}}$ and $\mu=\|\alpha \tilde{\boldsymbol{q}}-\tilde{\boldsymbol{p}}\|_{1}$. Then $\tilde{\boldsymbol{r}}_{T} \in \mathcal{P}_{\rho}$,

$$
\boldsymbol{\lambda}+\mu(-\boldsymbol{r})=\tilde{\boldsymbol{p}},
$$


and

$$
\lambda_{n} \boldsymbol{z}_{n}-\sum_{m \in c(n)} \lambda_{m} \boldsymbol{z}_{m}=\mathbf{0} \quad\left(n \in \mathcal{N}_{-T}\right)
$$

hold since $\boldsymbol{\lambda}$ is a positive multiple of the martingale probability measure $\tilde{\boldsymbol{q}}$. The equation (25) implies that $\lambda_{n} \boldsymbol{z}_{n}+\mu\left(-\tilde{r}_{n} \boldsymbol{z}_{n}\right)=p_{n} \boldsymbol{z}_{n}$ for $n \in \mathcal{N}_{T}$. Combining this with (26) shows that the dual problem of problem (17) is feasible. Then, by the weak duality theorem Lemma A.1 in Appendix, the optimal value of the problem is zero, meaning no $\rho$-arbitrage.

Note that Theorem 3.3 does not require $\boldsymbol{p}$ be in $\mathcal{P}_{\rho}$.

Let $\mathcal{M}$ be the set of probability measure under which $\left\{\boldsymbol{Z}_{t} \mid t \in[T]\right\}$ is a martingale process. The following corollary states a generalized version of the fundamental theorem Theorem 2.1 under the condition that $\boldsymbol{p} \in \operatorname{ri}_{\Pi}\left(\mathcal{P}_{\rho}\right)$.

Corollary 3.1. Suppose $\boldsymbol{p} \in \operatorname{ri}_{\Pi}\left(\mathcal{P}_{\rho}\right)$. Suppose that the system (13), (14) and (15) satisfy the Slater constraint qualification or $\mathcal{P}_{\rho}$ be a polytope. Then there is no $\rho$-arbitrage if and only if there is $\tilde{\boldsymbol{q}} \in \mathcal{M}$ such that $\tilde{\boldsymbol{q}}_{T} \in r i_{\Pi}\left(\mathcal{P}_{\rho}\right)$.

Proof. Note that the probability measure $\tilde{\boldsymbol{q}}$ whose existence was given by Theorem 3.1 satisfies $\tilde{\boldsymbol{q}}_{T} \in$ $\operatorname{ri}_{\Pi}\left(\mathcal{P}_{\rho}\right)$ when $\boldsymbol{p} \in \operatorname{ri}_{\Pi}\left(\mathcal{P}_{\rho}\right)$. See the Accessibility Lemma (3.2.11) of Stoer and Witzgall [21] or Theorem 2.33 of Rockafellar and Wets [22]. Then the assertion is straightforward from the two theorems.

\section{Pricing European-Type Contingent Claims}

In this section, we consider the pricing problem of European-type contingent claims. European-type contingent claim is a claim which provides the holder with the right to receive payoff at maturity date $T$. Among examples is a European call option that gives the holder the right to buy the security at price $K$ at maturity date $T$. The value of the option at $T$ is then given by $\max \left\{0, S_{T}-K\right\}$, where $S_{T}$ denotes the security price at $T$. We begin with considering the problem of hedging the risk arising from the claims.

Definition 4.1 ( $\rho$-Hedging). Let $y_{n}$ denote the value of a contingent claim $Y$ at each terminal node $n \in \mathcal{N}_{T}$, and let $c_{n}$ denote the relative value of $y_{n}$ with respect to the numeraire $s_{n}^{0}$, i.e., $c_{n}:=y_{n} / s_{n}^{0}$ for $n \in \mathcal{N}$. A self-financing trading strategy $\boldsymbol{\theta}$ is the $\rho$-hedging strategy of $Y$ iff

1) the expected value of the portfolio consisting of $\theta_{n}^{j}$ units of security $j$ for $j=0, \ldots, J$ and -1 unit of the claim is nonnegative, i.e., $\sum_{n \in \mathcal{N}_{T}} p_{n}\left(\boldsymbol{z}_{n}^{\top} \boldsymbol{\theta}_{n}-c_{n}\right) \geq 0$, and 
2) the future wealth of the portfolio is acceptable with respect to the coherent risk measure $\rho$, i.e., $\rho\left(\left(\boldsymbol{z}_{n}^{\top} \boldsymbol{\theta}_{n}-c_{n}\right)_{n \in \mathcal{N}_{T}}\right) \leq 0$.

Note that the second condition $\rho\left(\left(\boldsymbol{z}_{n}^{\top} \boldsymbol{\theta}_{n}-c_{n}\right)_{n \in \mathcal{N}_{T}}\right) \leq 0$ holds if and only if

$$
\sum_{n \in \mathcal{N}_{T}} \pi_{n}\left(\boldsymbol{z}_{n}^{\top} \boldsymbol{\theta}_{n}-c_{n}\right) \geq 0, \quad \text { for all } \boldsymbol{\pi} \in \mathcal{P}_{\rho}
$$

\subsection{Computation of Upper and Lower Bounds of Price}

We start this subsection with considering the seller's pricing problem of a European-type contingent claim using the $\rho$-hedging.

The value $c_{n}$ is the future cashflow that the seller of the contingent claim $Y$ pays to the buyer at each terminal node $n \in \mathcal{N}_{T}$. An upper bound of the price of the claim is then the minimum cost for the seller to $\rho$-hedge this claim, which is mathematically given by the optimal value of the following problem.

$$
\begin{array}{|lll}
\text { inf } & \boldsymbol{z}_{0}^{\top} \boldsymbol{\theta}_{0}, & \\
\text { subject to } & \boldsymbol{z}_{n}^{\top}\left(\boldsymbol{\theta}_{n}-\boldsymbol{\theta}_{a(n)}\right)=0, & \left(n \in \mathcal{N}_{-0}\right), \\
& \sum_{n \in \mathcal{N}_{T}} \pi_{n} \boldsymbol{z}_{n}^{\top} \boldsymbol{\theta}_{n} \geq \sum_{n \in \mathcal{N}_{T}} \pi_{n} c_{n}, \quad\left(\boldsymbol{\pi} \in \mathcal{P}_{\rho}\right), \\
& \sum_{n \in \mathcal{N}_{T}} p_{n} \boldsymbol{z}_{n}^{\top} \boldsymbol{\theta}_{n} \geq \sum_{n \in \mathcal{N}_{T}} p_{n} c_{n} . &
\end{array}
$$

Throughout this section, we assume that $\boldsymbol{p} \in \mathcal{P}_{\rho}$, then the last constraint is redundant and can be omitted. We assume that the Slater constraint qualification be satisfied. Then the uniform LP duality in Appendix holds with the dual problem

$$
\begin{array}{|lll}
\sup & \sum_{\pi \in \mathcal{P}_{\rho}} \mu_{\pi}\left(\sum_{n \in \mathcal{N}_{T}} \pi_{n} c_{n}\right), & \\
\text { subject to } & \lambda_{0}=1, & \\
& \lambda_{n} \boldsymbol{z}_{n}=\sum_{m \in c(n)} \lambda_{m} \boldsymbol{z}_{m}, & \left(n \in \mathcal{N}_{-T}\right), \\
& \lambda_{n} \boldsymbol{z}_{n}=\sum_{\pi \in \mathcal{P}_{\rho}} \mu_{\pi} \pi_{n} \boldsymbol{z}_{n}, & \left(n \in \mathcal{N}_{T}\right), \\
& \mu_{\pi} \geq 0, & \left(\boldsymbol{\pi} \in \mathcal{P}_{\rho}\right), \\
& \left|\left\{\boldsymbol{\pi} \in \mathcal{P}_{\rho} \mid \mu_{\pi}>0\right\}\right|<\infty . &
\end{array}
$$

Note that the equality constraints imply that $\sum_{\pi \in \mathcal{P}_{\rho}} \mu_{\pi}=1$ and $\lambda_{n}=\sum_{\pi \in \mathcal{P}_{\rho}} \mu_{\pi} \pi_{n}$, for $n \in \mathcal{N}_{T}$. Then letting

$$
\tilde{q}_{n}:= \begin{cases}\lambda_{n} & \text { for } n \in \mathcal{N}_{-T} \\ \sum_{\pi \in \mathcal{P}_{\rho}} \mu_{\pi} \pi_{n} & \text { for } n \in \mathcal{N}_{T}\end{cases}
$$

and $\boldsymbol{c}=\left(c_{n}\right)_{n \in \mathcal{N}_{T}}$, and using the convexity of $\mathcal{P}_{\rho}$, the dual problem is equivalent to

$$
\begin{array}{|ll}
\sup & \tilde{\boldsymbol{q}}_{T}^{\top} \boldsymbol{c}, \\
\operatorname{subject} \text { to } & \tilde{q}_{0}=1, \\
& \tilde{q}_{n} \boldsymbol{z}_{n}=\sum_{m \in c(n)} \tilde{q}_{m} \boldsymbol{z}_{m}, \quad\left(n \in \mathcal{N}_{-T}\right), \\
& \tilde{\boldsymbol{q}}_{T} \in \mathcal{P}_{\rho},
\end{array}
$$

which is further rewritten as

$$
\begin{array}{|ll}
\sup & \mathbb{E}_{\tilde{\boldsymbol{q}}_{T}}(\boldsymbol{c}) \\
\text { subject to } & \tilde{\boldsymbol{q}}_{T} \in \mathcal{M}_{T} \cap \mathcal{P}_{\rho}
\end{array}
$$


where $\mathcal{M}_{T}:=\left\{\tilde{\boldsymbol{q}}_{T} \mid \tilde{\boldsymbol{q}} \in \mathcal{M}\right\}$.

Lemma 4.1. Suppose $\boldsymbol{p} \in \mathcal{P}_{\rho}$ and that the constraints of (27) satisfy the Slater constraint qualification. Then the upper bound of claim Y's price is given by the optimal value of the problem (30).

We obtain the same result without assuming the Slater constraint qualification when $\mathcal{P}_{\rho}$ is a polytope.

On the other hand, the buyer pays $y_{0}$ in return for a promise of payments $y_{n}$ at each terminal node $n \in \mathcal{N}_{T}$, and the exposure of buyer is then $-y_{n}$. Under the assumption of no $\rho$-arbitrage, the cost for $\rho$-hedging this position is no less than the initial value of this position. Then the buyer's problem for obtaining the largest initial cost for $\rho$-hedging the risk may be modeled as

$$
\begin{array}{|lll}
\text { inf } & \boldsymbol{z}_{0}^{\top} \boldsymbol{\theta}_{0}, & \\
\text { subject to } & \boldsymbol{z}_{n}^{\top}\left(\boldsymbol{\theta}_{n}-\boldsymbol{\theta}_{a(n)}\right)=0, & \left(n \in \mathcal{N}_{-0}\right), \\
& \sum_{n \in \mathcal{N}_{T}} \pi_{n} \boldsymbol{z}_{n}^{\top} \boldsymbol{\theta}_{n} \geq-\sum_{n \in \mathcal{N}_{T}} \pi_{n} c_{n}, \quad\left(\boldsymbol{\pi} \in \mathcal{P}_{\rho}\right), \\
& \sum_{n \in \mathcal{N}_{T}} p_{n} \boldsymbol{z}_{n}^{\top} \boldsymbol{\theta}_{n} \geq-\sum_{n \in \mathcal{N}_{T}} p_{n} c_{n} . &
\end{array}
$$

Again assuming the Slater constraint qualification, we obtain the dual problem:

$$
\mid \begin{array}{ll}
\sup & \mathbb{E}_{\tilde{\boldsymbol{q}}_{T}}(-\boldsymbol{c}) \\
\text { subject to } & \tilde{\boldsymbol{q}}_{T} \in \mathcal{M}_{T} \cap \mathcal{P}_{\rho}
\end{array}
$$

which is equivalent to

$$
\mid \begin{array}{ll}
-\inf & \mathbb{E}_{\tilde{\boldsymbol{q}}_{T}}(\boldsymbol{c}) \\
\text { subject to } & \tilde{\boldsymbol{q}}_{T} \in \mathcal{M}_{T} \cap \mathcal{P}_{\rho} .
\end{array}
$$

The optimal value of (33) gives the upper bound of initial value $-c_{0}$. Then we have the following lemma.

Lemma 4.2. Suppose $\boldsymbol{p} \in \mathcal{P}_{\rho}$ and that the constraints of (31) satisfy the Slater constraint qualification. Then the lower bound of claim $Y$ 's price is given by the optimal value of the problem (33).

Combining the two lemmas we obtain the following theorem.

Theorem 4.1. Suppose $\boldsymbol{p} \in \mathcal{P}_{\rho}$. Suppose either that the constraints of (27) and (31) satisfy the Slater constraint qualification or that $\mathcal{P}_{\rho}$ be a polytope. Then the lower and upper bounds of claim $Y$ 's price is given by

$$
\inf \left\{\mathbb{E}_{\tilde{\boldsymbol{q}}_{T}}(\boldsymbol{c}) \mid \tilde{\boldsymbol{q}}_{T} \in \mathcal{M}_{T} \cap \mathcal{P}_{\rho}\right\} \quad \text { and } \sup \left\{\mathbb{E}_{\tilde{\boldsymbol{q}}_{T}}(\boldsymbol{c}) \mid \tilde{\boldsymbol{q}}_{T} \in \mathcal{M}_{T} \cap \mathcal{P}_{\rho}\right\}
$$

\section{Numerical Illustration}

We provide some illustrative examples of pricing a European-type contingent claim in a simple setting. 


\section{$5.1 \beta$-CVaR Pricing}

Below are three well-known coherent risk measures $\rho(\boldsymbol{x})$ for net worth $\boldsymbol{x}$ and their corresponding set of probability measure $\mathcal{P}_{\rho}$. Here we write $\Pi_{+}:=\left\{\boldsymbol{\pi} \in \mathbb{R}^{n_{T}} \mid \boldsymbol{\pi} \geq \mathbf{0}, \boldsymbol{e}^{\top} \boldsymbol{\pi}=1\right\}$ and $[x]^{+}=\max \{x, 0\}$.

1) Maximum loss:

$$
\begin{aligned}
& \rho(\boldsymbol{x})=\max \left\{-x_{n} \mid n \in \mathcal{N}_{T}\right\}, \\
& \mathcal{P}_{\rho}=\Pi_{+} .
\end{aligned}
$$

2) Mean absolute semi-deviation:

$$
\begin{aligned}
& \rho(\boldsymbol{x})=\sum_{n \in \mathcal{N}_{T}} p_{n}\left(-x_{n}\right)+\lambda \sum_{n \in \mathcal{N}_{T}} p_{n}\left[x_{n}-\sum_{n \in \mathcal{N}_{T}} p_{n} x_{n}\right]^{+} \text {with } \lambda \in(0,1 / 2), \\
& \mathcal{P}_{\rho}=\left\{\boldsymbol{\pi} \in \Pi_{+} \mid(\lambda+1) /(2 \lambda+1) \boldsymbol{p} \leq \boldsymbol{\pi} \leq(1+\lambda) \boldsymbol{p}\right\} .
\end{aligned}
$$

3) Conditional value-at-risk (CVaR):

$$
\begin{aligned}
& \rho(\boldsymbol{x})=\min \left\{\alpha+1 /(1-\beta) \sum_{n \in \mathcal{N}_{T}} p_{n}\left[-x_{n}-\alpha\right]^{+} \mid \alpha \in \mathbb{R}\right\}, \\
& \mathcal{P}_{\rho}=\left\{\boldsymbol{\pi} \in \Pi_{+} \mid \boldsymbol{\pi} \leq \boldsymbol{p} /(1-\beta)\right\} .
\end{aligned}
$$

The above three measures satisfies the condition $\boldsymbol{p} \in \mathcal{P}_{\rho}$ in Theorem 3.1. Furthermore, all of the $\mathcal{P}_{\rho}$ 's above are polytopes, hence Theorem 3.1 and Theorem 4.1 hold without the Slater constraint qualification. CVaR has a parameter $\beta$ ranging in $[0,1)$. The corresponding $\mathcal{P}_{\rho}$ coincides with $\Pi_{+}$when $\beta \geq 1-\min \left\{p_{n} \mid\right.$ $\left.n \in \mathcal{N}_{T}\right\}$, and becomes smaller as $\beta$ decreases. It is worth emphasizing here that the use of CVaR has advantage in that the bounding problems (34) result in linear optimizations, for which a series of parametric solutions can be efficiently obtained. Therefore we use CVaR as the risk measure $\rho$ in the following example.

Take a single period example where the scenario tree has three terminal nodes (i.e., $T=1, \mathcal{N}_{T}=$ $\{1,2,3\})$. The sets of $\mathcal{M}_{T}$ and $\mathcal{P}_{\rho}$ are then shown as in Figure 1 where $\mathcal{M}_{T}$ is the line segment connecting $\mathrm{A}$ and $\mathrm{B}, \mathcal{P}_{\rho}$ is the hexagon and $\mathcal{M}_{T} \cap \mathcal{P}_{\rho}$ is the line segment connecting $\mathrm{C}$ and $\mathrm{D}$.

When $\beta \geq 1-\min _{n \in \mathcal{N}_{t}} p_{n}$, one has $\mathcal{P}_{\rho}=\Pi_{+}$, the no- $\beta$-CVaR-arbitrage interval coincides with the noarbitrage interval that calculated by the fundamental theorem of asset price, i.e., Theorem 2.1. The set $\mathcal{P}_{\rho}$ becomes smaller as $\beta$ decreases from 1 to 0 and the interval (34) becomes smaller as well.

In order to illustrate how the upper and lower bounds shrink, let us provide a numerical example. Figure 2 depicts an uncertain structure of single period market model, where three basic assets and a European call option are traded and four possible future states are considered at each branching. In the figure, the values of the three basic assets are indicated in the boxes attached to each circle representing a state, while the value of the call option is indicated just below each box in italic. Figure 3 shows the 


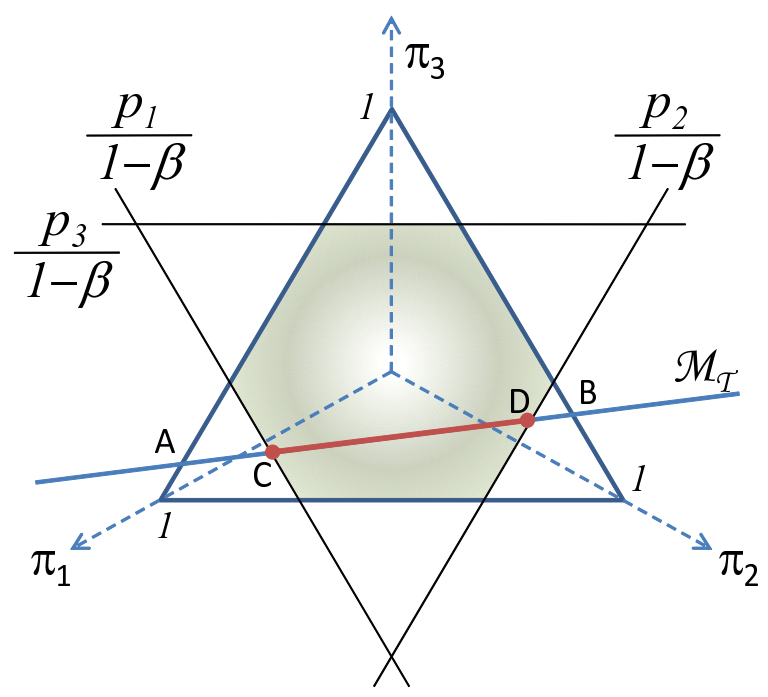

Figure 1: $\mathcal{M}_{T} \cap \mathcal{P}_{\rho}$ associated with $\beta$-CVaR

$\rho$-arbitrage interval of the call option, calculated by the $\beta$-CVaR model for $\beta$ from 0.70 to 0.416667 , where UB stands for the upper bound, and LB stands for the lower one.

The price interval provided by the ordinary no-arbitrage theory is $[6.25,15.7895]$, while that of the extended one shrinks as the value of $\beta$ decreases. When $\beta=0.416667$, the no $\rho$-arbitrage price is unique and 12.7232, which means that if the European call option is traded at 12.7232, the buyer and seller face with the same amount of risk in terms of $\beta$-CVaR. In this sense, this value can be considered as a fair price of this option.

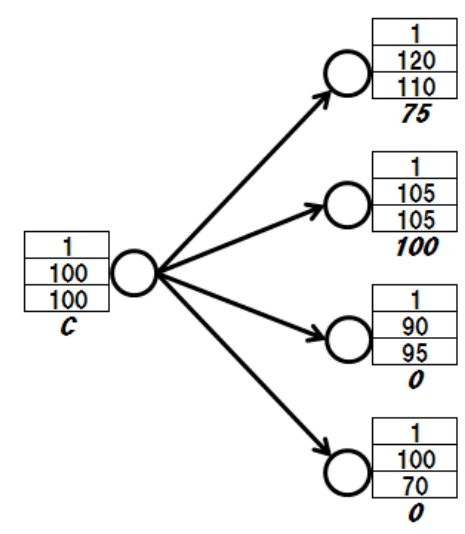

Figure 2: An Example of Single Period Incomplete Market Model 


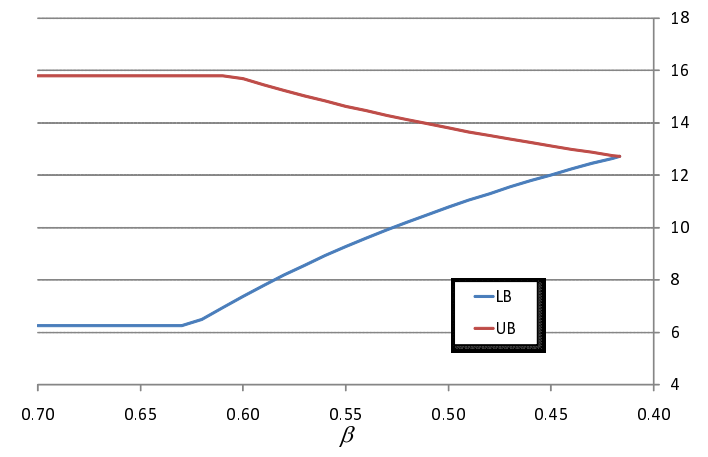

Figure 3: Price Bound Shrinking via CVaR Model: Horizontal axis is the value of $\beta$ in CVaR. Two bounds converge to 12.7232 at $\beta=0.416667$.

\subsection{Pricing with Non-polyhedral Probability Sets and a Robust Optimization Viewpoint}

In addition to the previous examples where the probability set $\mathcal{P}_{\rho}$ is a polytope, we give two nonpolyhedral examples of $\mathcal{P}_{\rho}$.

Relative Entropy Restriction Suppose $\boldsymbol{p}>\mathbf{0}$ and let $I(\boldsymbol{q} ; \boldsymbol{p}):=\sum_{n \in \mathcal{N}_{T}} q_{n} \ln \frac{q_{n}}{p_{n}}$, which is the socalled relative entropy of $\boldsymbol{q}$ with respect to $\boldsymbol{p}$. Let the probability set $\mathcal{P}_{\rho}$ be given by

$$
\mathcal{P}_{\rho}:=\left\{\boldsymbol{q} \in \Pi_{+} \mid I(\boldsymbol{q} ; \boldsymbol{p}) \leq C\right\}
$$

where $C$ is a positive constant. The corresponding problems (32) and (33) are still convex optimization problems because $I(\cdot ; \boldsymbol{p})$ is a convex function on the unit simplex $\Pi_{+}$.

Ellipsoidal Distance Restriction For a positive definite matrix $G$ let

$$
E_{G}(\boldsymbol{q} ; \boldsymbol{p}):=\left\{(\boldsymbol{q}-\boldsymbol{p})^{\top} G(\boldsymbol{q}-\boldsymbol{p})\right\}^{1 / 2}
$$

and

$$
\mathcal{P}_{\rho}:=\left\{\boldsymbol{q} \in \Pi_{+} \mid E_{G}(\boldsymbol{q} ; \boldsymbol{p}) \leq 1\right\} .
$$

When $G$ is a diagonal matrix of diagonal elements $\left(g_{n}\right)_{n \in \mathcal{N}_{T}}, E_{G}(\boldsymbol{q} ; \boldsymbol{p})=\left\{\sum_{n \in \mathcal{N}_{T}} g_{n}\left(q_{n}-p_{n}\right)^{2}\right\}^{1 / 2}$, and it is readily seen that the resulting optimization problem is a second order cone optimization.

In the above two examples, $\boldsymbol{p}$ lies in $\mathrm{ri}_{\Pi} \mathcal{P}_{\rho}$, which ensures the generalized version of the fundamental theorem, Corollary 3.1. At the same time, this construction enables one to see the pricing as a robust optimization (see, e.g., [18]). Namely, the constraints such as $I(\boldsymbol{q} ; \boldsymbol{p}) \leq C$ and $E_{G}(\boldsymbol{q} ; \boldsymbol{p}) \leq 1$ prevent the 


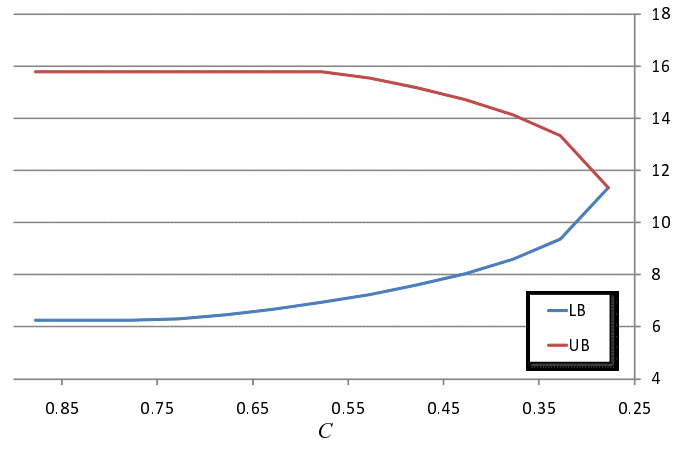

Figure 4: Price Bound Shrinking via Entropy Model: Horizontal axis is the value of $C$ in (35). Two bounds converge to 11.3258 at $C=0.277585$.

probability $\boldsymbol{q}$ from deviating from the nominal probability $\boldsymbol{p}$, and define convex uncertainty sets. With these sets, the hedging problems (27) and (31) for sellers and buyers can be regarded as robust hedge problems, where the hedging portfolio would be robust against the uncertainty of probability under which expected cash flows of the claim are evaluated. Figure 4 illustrates the upper and lower bounds of the option price in Figure 2 for the case of $\mathcal{P}_{\rho}$ in (35). The two bounds are observed to converge to a single value, 11.3258, which is, however, different from the value given by CVaR.

\subsection{A Real Data Example}

The methodology developed thus far can apply to incomplete market settings or a situation where one wants to evaluate nonmarketable assets. Those structures are often too complicated to obtain analytical price formulae. We take an Asian put option on a security listed on the Nikkei225 index as an example. The tree is of depth 3 and each node has 10 child nodes, so that the terminal nodes amounts to 1000 . Three risky securities in the Nikkei225 index are used to $\rho$-hedge the option on the first security and the rate of return of the numeraire is set to be zero. The security prices on each node are computed by multiplying ten historical returns by prices on its parent node. Figure 5 shows that the pricing bounds contracts from the ordinary arbitrage interval $[2.62,31.91]$ as the parameter values decrease. The entropy model achieves almost a single value while a small gap remains for the CVaR model. Once a coherent risk measure is chosen, these bounds are independent of preference or utility function of investors. This is a nice property, especially when a fair price of a nonmarketable asset is needed. We should add that each of the nonlinear convex optimization problem was solved within one second on a laptop computer. 

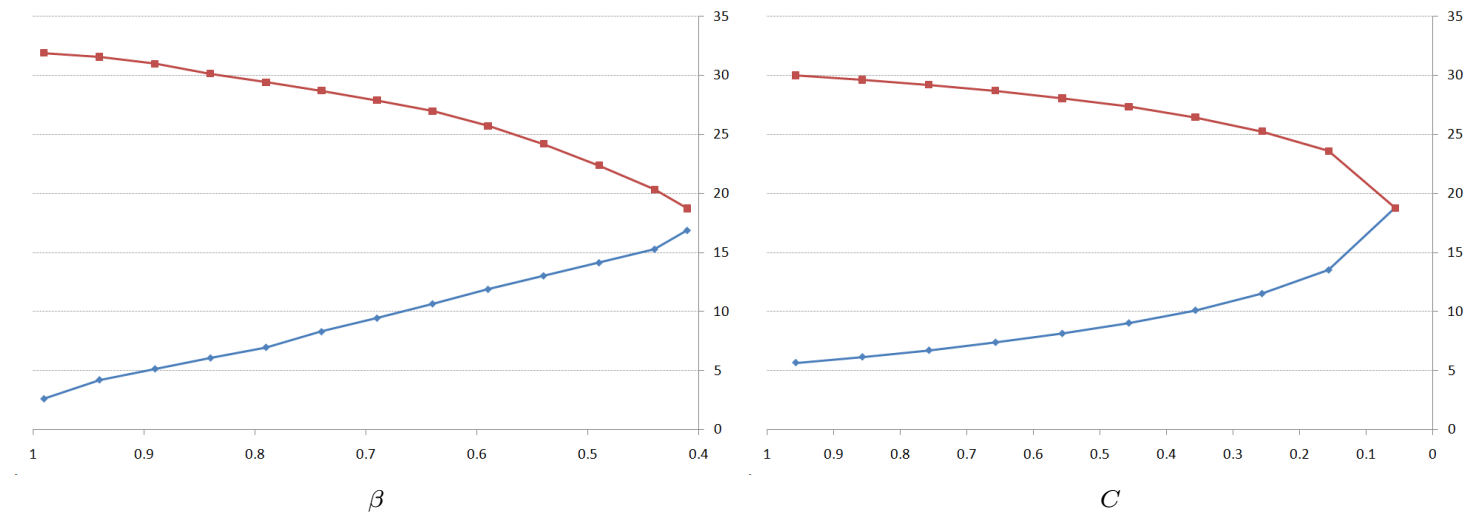

Figure 5: Price Bounds of an Asian Put Option: CVaR model (Left) and Entropy model (Right)

\section{Conclusion}

In this paper, we investigate an optimization approach for tightening the bounds of the price of Europeantype contingent claims in discrete-time and discrete-state incomplete market models.

In contrast to the traditional fundamental theorem of finance which is described in the perfect hedge framework as in [10], this paper examines how the martingale probability relates to the hedging strategy based on coherent risk measures. We showed that the existence of a restricted martingale probability is equivalent to the non-existence of a generalized arbitrage through the duality theory of semi-infinite linear optimization.

We also showed that upper and lower bounds of a contingent claim price can be obtained as the optimal values of super-replicating problems for sellers and buyers, respectively. This formulation is more realistic than the perfect replication in the traditional setting since both sellers and buyers are forced to take some risk of a positive loss in actual and obviously incomplete markets. If the coherent risk measure is adequately described with a parameter such as $\beta$ of CVaR, one can fill up the gap between the bounds by tuning the parameter. Moreover, as shown in the numerical examples, a wider range of contingent claims such as exotic options can be priced by the proposed method. For further details of the tree construction, see a series of works of Hoyland and Wallace, e.g., [23].

\section{References}

1. Schouten, W.: Levy process in finance: pricing financial derivatives. Wiley, USA (2003) 
2. Heston, S.L.: A Closed-Form Solution for Options with Stochastic Volatility with Applications to Bond and Currency Options. Review of Financial Studies. 6, 327-344 (1993)

3. Lo, A.: Semi-parametric upper bounds for option prices and expected payoffs. Journal of Financial Economics. 19, 373-387 (1987)

4. Bertsimas, D., Popescu, I.: On the relation between option and stock prices: A convex optimization approach. Operations Research. 50, 358-374 (2002)

5. Gotoh, J., Konno, H.: Bounding option prices by semidefinite programming: A cutting plane algorithm. Operations Research. 48, 665-678 (2002)

6. Lesserre, J.B., Prieto-Rumeau, T., Zervos, M.: Pricing a class of exotic options via moments and SDP relaxations. Mathematical Finance. 16, 469-494 (2006)

7. Cox, J.C., Ross, S.A., Rubinstein, M.: Option pricing: Simplified approach. Journal of Financial Economics. 7, 229-263 (1979)

8. Hull, J., White, A.: Bond option pricing based on a model for the evolution of bond price. Advances in Futures and Options Research. 6, 1-13 (1993)

9. Pliska, S.R.: Introduction to mathematical finance -Discrete time models-. Blackwell Publishers, USA (1997)

10. King, A.: Duality and martingales: a stochastic programming perspective on contingent claims. Mathematical Programming, Ser. B. 91, 543-562 (2002)

11. Ritchken, P.: On option pricing bounds. Journal of Finance. 40, 1219-1233 (1985)

12. Ritchken, P., Kuo, S.: On stochastic dominance and decreasing absolute risk averse option pricing bounds. Management Science. 35, 51-59 (1989)

13. Basso, A., Pianca, P.: Decreasing absolute risk aversion and option pricing bounds. Management Science. 43, 206-216 (1997)

14. King, A., Pennanen, T.: Arbitrage pricing of American contingent claims in incomplete markets - a convex optimization approach. Working paper, Helsinki School of Economics, (2005)

15. Jaschke, S., Küchler, U.: Coherent risk measures and good-deal bounds. Finance and Stochastics. 5, 181-200 (2001) 
16. Nakano, Y.: Minimizing coherent risk measures of shortfall in discrete-time models with cone constraints. Applied Mathematical Finance. 10, 163-181 (2003)

17. Rockafellar, T.R., Uryasev, S.: Conditional value-at-risk for general loss distributions. J. Banking and Finance. 26, 1443-1471 (2002)

18. Bertsimas, D., Brown, D.B., Caramanis, C.: Theory and applications of robust optimization. Discussion paper, MIT, (2008)

19. Harrison, J.M., Kreps, D.M.: Martingales and arbitrage in multiperiod securities markets. Journal of Economic Theory. 20, 381-408 (1979)

20. Artzner, P., Delbaen, F., Eber, J-M. and Heath, D.: Coherent measures of risk. Mathematical Finance. 9, 203-22 (1999)

21. Stoer, J., Witzgall, C.: Convexity and optimization in finite dimension I. Springer, Berlin (1970)

22. Rockafellar, R.T., Wets, R. J-B.: Variational Analysis. Springer, Berlin (1998)

23. Holyand, K., Wallace, S.W.: Generating scenario trees for multi-stage decision problems. Management Science. 47, 295-307 (2001)

24. Glashoff, K., Gustafson, S.-A.: Linear Optimization and Approximation. Springer-Verlag, Berlin (1983)

25. Duffin, R.J., Jeroslow, R.G., Karlovitz, L.A.: Duality in semi-infinite linear programming, SemiInfinite Programming and Applications, Springer-Verlag, Berlin (1983)

26. Gehner, K.R.: Necessary and sufficient optimality conditions for the Fritz John problem with linear equality constraints. SIAM Journal on Control. 12, 140-149 (1974)

\section{A Appendix}

Let $\bar{S}$ be a finite subset of $\mathbb{R}^{n+1}$ and $\bar{T}$ be a compact subset of $\mathbb{R}^{n+1}$. A semi-infinite linear optimization problem defined by $\bar{S}, \bar{T}$ and $\boldsymbol{c} \in \mathbb{R}^{n}$ is the problem

$$
\begin{array}{|lll}
\text { inf } & \boldsymbol{c}^{\top} \boldsymbol{x} & \\
\text { subject to } & \boldsymbol{s}^{\top} \boldsymbol{x}=s_{0} & \left(\left(\boldsymbol{s}, s_{0}\right) \in \bar{S}\right) \\
& \boldsymbol{t}^{\top} \boldsymbol{x} \geq t_{0} & \left(\left(\boldsymbol{t}, t_{0}\right) \in \bar{T}\right) .
\end{array}
$$

The dual problem is 


$$
\begin{array}{|ll}
\sup & \sum_{\left(s, s_{0}\right) \in \bar{S}} \lambda_{\left(s, s_{0}\right)} s_{0}+\sum_{\left(t, t_{0}\right) \in \bar{T}} \mu_{\left(t, t_{0}\right)} t_{0} \\
\text { subject to } & \sum_{\left(s, s_{0}\right) \in \bar{S}} \lambda_{\left(s, s_{0}\right)} s+\sum_{\left(t, t_{0}\right) \in \bar{T}} \mu_{\left(t, t_{0}\right)} \boldsymbol{t}=\boldsymbol{c} \\
& \left.\mu_{\left(t, t_{0}\right)} \geq 0\left(\boldsymbol{t}, t_{0}\right) \in \bar{T}\right) \\
& \left|\left\{\left(\boldsymbol{t}, t_{0}\right) \in \bar{T} \mid \mu_{\left(t, t_{0}\right)}>0\right\}\right|<\infty .
\end{array}
$$

Let $v(P)$ and $v(D)$ denote the optimal objective function value of $(P)$ and $(D)$, respectively. Then the weak duality theorem holds. See Theorem (18) in Glashoff and Gustafson [24] for the proof.

\section{Lemma A.1.}

$$
v(D) \leq v(P) .
$$

The pair $(P)$ and $(D)$ is said to yield uniform LP duality if for each $\boldsymbol{c} \in \mathbb{R}^{n}$ exactly one of the following cases holds:

(i) $v(P)=-\infty$ and $(D)$ is infeasible.

(ii) $v(D)=\infty$ and $(P)$ is infeasible.

(iii) Both $(P)$ and $(D)$ are infeasible.

(iv) Both $(P)$ and $(D)$ are feasible and $v(P)=v(D)$, which is attained by some feasible solution of $(D)$. We say that primal problem $(P)$ satisfies the Slater constraint qualification when there is a vector $\hat{\boldsymbol{x}} \in \mathbb{R}^{n}$ such that

$$
\begin{array}{cc}
\boldsymbol{s}^{\top} \hat{\boldsymbol{x}}=s_{0} & \left(\left(\boldsymbol{s}, s_{0}\right) \in \bar{S}\right) \\
\boldsymbol{t}^{\top} \hat{\boldsymbol{x}}>t_{0} & \left(\left(\boldsymbol{t}, t_{0}\right) \in \bar{T}\right) .
\end{array}
$$

Combining Theorem 2.2 and Theorem 3.2 of Duffin, Jeroslow and Karlovitz [25] we have the following lemma. See also Gehner [26].

Lemma A.2. If the primal problem $(P)$ satisfies the Slater constraint qualification, the problem pair $(P)$ and $(D)$ yields uniform LP duality.

When $s_{0}=0$ for all $\left(\boldsymbol{s}, s_{0}\right) \in \bar{S}$ and $t_{0}=0$ for all $\left(\boldsymbol{t}, t_{0}\right) \in \bar{T}$, the primal and dual problems reduce to

$$
\begin{array}{|lll}
\inf & \boldsymbol{c}^{\top} \boldsymbol{x} & \\
\text { subject to } & \boldsymbol{s}^{\top} \boldsymbol{x}=0 \quad(\boldsymbol{s} \in S) \\
& \boldsymbol{t}^{\top} \boldsymbol{x} \geq 0 \quad(\boldsymbol{t} \in T)
\end{array}
$$

and

$$
\begin{array}{|ll}
\sup & 0 \\
\operatorname{subject} \text { to } & \sum_{s \in S} \lambda_{s} s+\sum_{t \in T} \mu_{t} \boldsymbol{t}=\boldsymbol{c} \\
& \mu_{t} \geq 0 \\
& \left|\left\{\boldsymbol{t} \in T \mid \mu_{t}>0\right\}\right|<\infty
\end{array} \quad(\boldsymbol{t} \in T)
$$


where $S$ is a finite subset of $\mathbb{R}^{n}$ and $T$ is a compact subset of $\mathbb{R}^{n}$. The Slater constraint qualification is the existence of $\hat{\boldsymbol{x}}$ such that

$$
\begin{array}{cc}
\boldsymbol{s}^{\top} \hat{\boldsymbol{x}}=0 & (\boldsymbol{s} \in S) \\
\boldsymbol{t}^{\top} \hat{\boldsymbol{x}}>0 & (\boldsymbol{t} \in T) .
\end{array}
$$

By Lemma A.2 we see that the problem pair $\left(P_{0}\right)$ and $\left(D_{0}\right)$ yields uniform LP duality when $\left(P_{0}\right)$ satisfies the Slater constraint qualification. Then we obtain the following lemma.

Lemma A.3. Suppose $\left(P_{0}\right)$ satisfy the Slater constraint qualification. Then $v\left(P_{0}\right)=0$ if and only if $\left(D_{0}\right)$ has a feasible solution.

Lemma A.4. Let $\boldsymbol{p}=\left(p_{1}, \ldots, p_{m}\right)^{\top}$ and $\boldsymbol{q}=\left(q_{1}, \ldots, q_{m}\right)^{\top}$ be nonnegative real vectors of $\mathbb{R}^{m}$ such that 1) $\boldsymbol{e}^{\top} \boldsymbol{p}=\boldsymbol{e}^{\top} \boldsymbol{q}=1$

2) $q_{n}>0$ whenever $p_{n}>0$,

where $\boldsymbol{e} \in \mathbb{R}^{m}$ is a vector of ones. Then for any positive $\varepsilon$ there is a positive $\alpha$ such that

$$
\left\|\frac{\alpha \boldsymbol{q}-\boldsymbol{p}}{\|\alpha \boldsymbol{q}-\boldsymbol{p}\|_{1}}-\boldsymbol{q}\right\|<\varepsilon
$$

holds, where $\|\cdot\|_{1}$ denotes the 1 -norm and $\|\cdot\|$ denotes any norm of $\mathbb{R}^{m}$.

Proof. We will show that any real number $\alpha$ such that

$$
\alpha>\max \left\{\frac{\|\boldsymbol{p}-\boldsymbol{q}\|}{\varepsilon}+1, \max \left\{\frac{p_{n}}{q_{n}} \mid p_{n}>0\right\}\right\}
$$

meets the condition of the lemma. Note first that $\alpha q_{n}-p_{n} \geq 0$ since $\alpha>p_{n} / q_{n}$ whenever $p_{n}>0$, and hence $\|\alpha \boldsymbol{q}-\boldsymbol{p}\|_{1}=\boldsymbol{e}^{\top}(\alpha \boldsymbol{q}-\boldsymbol{p})=\alpha-1$. Therefore by the choice of $\alpha$ we see

$$
\begin{aligned}
\left\|\frac{\alpha \boldsymbol{q}-\boldsymbol{p}}{\|\alpha \boldsymbol{q}-\boldsymbol{p}\|_{1}}-\boldsymbol{q}\right\| & =\frac{1}{\|\alpha \boldsymbol{q}-\boldsymbol{p}\|_{1}}\|\alpha \boldsymbol{q}-\boldsymbol{p}-\| \alpha \boldsymbol{q}-\boldsymbol{p}\left\|_{1} \boldsymbol{q}\right\| \\
& =\frac{1}{\alpha-1}\|\alpha \boldsymbol{q}-\boldsymbol{p}-(\alpha-1) \boldsymbol{q}\| \\
& =\frac{1}{\alpha-1}\|\boldsymbol{q}-\boldsymbol{p}\|<\varepsilon .
\end{aligned}
$$

